\title{
Combined Bayesian Estimates for the Equicorrelation Coefficient
}

\author{
Marlos A. G. Viana * \\ The University of Illinois at Chicago
}

Key words and phrases: Bayesian estimation, corneal topography

AMS 1991 Mathematics Subject Classifications: 62F15, 62H20

\begin{abstract}
Combined Bayesian estimates for equicorrelation covariance matrices are considered. The case of a common equicorrelation $\rho$ and possibly different standard deviations $\sigma_{1}, \ldots, \sigma_{k}$ among $\mathrm{k}$ experimental groups is examined first, and the Bayesian estimation of $\left(\rho, \sigma_{1}, \ldots, \sigma_{k}\right)$ is discussed. Secondly, under the assumption of a common standard deviation and possibly different equicorrelations, the Bayesian estimation of $\left(\rho_{1}, \ldots, \rho_{k}, \sigma\right)$ is considered.
\end{abstract}

\section{Introduction}

Symmetric covariance models are naturally appropriate to model corneal curvature maps (keratometry) $\mathbf{X}$, consisting of curvature measurements $X_{1}, \ldots, X_{p}$. Of particular interest is the equicorrelation model in which $\operatorname{Var}\left(X_{1}\right)=\ldots=\operatorname{Var}\left(X_{p}\right)=\sigma^{2}$ and $\rho$ is the common correlation between curvatures of any two distinct measurement points. The objective of this paper is to obtain combined Bayesian estimates for the underlying covariance structure when samples of $\mathbf{X}$ are available from independent collaborating experiments.

An example of keratometry data is considered in the next section. The corresponding models, statistical analyses and applications are presented in Sections 3, 4 and 5, respectively. Additional comments and a summary are included in Section 6. Technical derivations are outlined in the Appendix.

Combined estimation of the the equicorrelation covariance structure is also important in applications dealing with bilateral units of analysis such as fellow eyes and fellow ears [Rosner $(1982,1984)]$, as well as in educational and psychological research (Olkin 1967). The model with common equicorrelation and possibly different variances among the $k$ studies fits the general framework of a common principal components model discussed in detail by Flury (1988). Similarly, the same model is related to the general problem of estimating $k$ covariance matrices with a common

${ }^{*}$ The research for this paper was supported in part by a grant from the Illinois Eye Fund to the Laboratory for Statistical Assessment of Diagnostic Tests, The University of Illinois at Chicago Eye and Ear Infirmary, 1855 West Taylor Street, Chicago, Illinois 60612. 
correlation matrix considered by Manly and Rayner (1987). A comprehensive discussion of methods of combining estimates of the standard correlation coefficient can be seen in Viana (1980), Hedges and Olkin (1985), and more recently in Han (1989).

\section{The Keratometry Data}

A typical curvature map $\mathbf{X}$ is based on about 20 concentric fields (rings or zones), which may be divided into 36 equally spaced semimeridians. The curvature is measured at each field and semimeridian so that each curvature mapping consists of 720 or more curvature measurements (Klyce and Wilson 1989, Hage 1976, Waring 1989). The present data are derived from $k=3$ collaborating studies designed to assess a computerized corneal topographer and are described in detail in Viana, Olkin and McMahon (1993). In each experiment the topography of a calibrated steel ball is replicated $N=10$ times and the results are considered as independent samples of $\mathbf{X}$.

Consider, specifically, the subregion of $\mathbf{X}$ specified by $p=4$ semimeridans 19,20,21,22 at the aperture corresponding to field 18 in each collaborating experiment $i=1,2,3$. The calibrated balls have curvatures, in diopters (D), 40.5 D, 42.5 D and $44.75 \mathrm{D}$. The observed correlation matrices $R_{i}$, and the vector $\mathbf{s}_{i}$ of sample standard deviations for experiment $i$, based on $N_{i}=10$ repeated mappings are :

$$
\begin{aligned}
& R_{1}=\left[\begin{array}{llll}
1.000 & 0.987 & 0.917 & 0.944 \\
0.987 & 1.000 & 0.889 & 0.917 \\
0.917 & 0.889 & 1.000 & 0.952 \\
0.944 & 0.917 & 0.952 & 1.000
\end{array}\right], \quad \mathbf{s}_{1}^{\prime}=(.061, .078, .049, .045), \\
& R_{2}=\left[\begin{array}{llll}
1.000 & 0.748 & 0.983 & 0.958 \\
0.748 & 1.000 & 0.833 & 0.796 \\
0.983 & 0.833 & 1.000 & 0.962 \\
0.958 & 0.796 & 0.962 & 1.000
\end{array}\right], \quad \mathbf{s}_{2}^{\prime}=(.098, .138, .082, .086), \\
& R_{3}=\left[\begin{array}{llll}
1.000 & 0.990 & 0.943 & 0.979 \\
0.990 & 1.000 & 0.943 & 0.979 \\
0.943 & 0.943 & 1.000 & 0.923 \\
0.979 & 0.979 & 0.923 & 1.000
\end{array}\right], \quad \mathbf{s}_{3}^{\prime}=(.124, .124, .144, .120)
\end{aligned}
$$

We observe that within each matrix, there is considerable homogeneity among the correlations and among the variances. However, between refractive powers, the variability in the data seems to differ. 


\section{The Models}

Suppose that a multivariate normal experiment on $\mathrm{p}$ random variables leads to the estimation of its covariance parameters under the equicorrelation structure

$$
\Sigma=\operatorname{Cov}(\mathbf{X})=\sigma^{2}\left[\begin{array}{cccc}
1 & \rho & \ldots & \rho \\
\rho & 1 & \ldots & \rho \\
\vdots & \vdots & \ldots & \vdots \\
\rho & \rho & \ldots & 1
\end{array}\right], \quad \frac{-1}{p-1}<\rho<1
$$

The covariance matrix contributes with two parameters; the intraclass or equicorrelation $\rho$ among any two distinct components of $\mathbf{X}$, and the variance parameter $\sigma^{2}=\operatorname{Var}\left(X_{j}\right), j=1, \ldots, p$. The experiment is then replicated by $k$ collaborating independent trials or studies, judged to be based on comparable experimental criteria. In this case, combined estimates of the joint covariance structure $\left(\rho_{1}, \sigma_{1}, \ldots, \rho_{k}, \sigma_{k}\right)$ become appropriate. Two basic models are considered in this article: The common-equicorrelation model assumes that $\rho_{1}=\ldots=\rho_{k}$ so that the purpose is jointly estimating $\left\{\rho, \sigma_{1}, \ldots, \sigma_{k}\right\}$ based on $S_{1}, \ldots, S_{k}$, the corresponding sample covariance matrices. The equal- variance equicorrelation model assumes that $\sigma_{1}=\ldots=\sigma_{k}$ and the joint estimation of $\left\{\rho_{1}, \ldots, \rho_{k}, \sigma\right\}$ is considered.

\section{Bayesian Estimates}

Consider $k$ independent sample covariance matrices $S_{1}, \ldots, S_{k}$ of dimension $p$ based on sample sizes $N_{1}, \ldots, N_{k}$ such that the distribution of $A_{i}=N_{i} S_{i}$ is Wishart with parameters $\Sigma_{i}$ and $n_{i}=N_{i}-1$, indicated by $A_{i} \sim W_{p}\left(\Sigma_{i}, n_{i}\right), i=1, \ldots, k$. Suppose also that each covariance matrix $\Sigma_{i}$ has the equicorrelation form (3.1), with corresponding variance $\sigma_{i}^{2}$ and equicorrelation $\rho_{i}$. The subscript $i$ indicates the experiments or studies being combined; its range is $1, \ldots, k$ and will be repeated in the remaining of the paper only when necessary. The following results are derived in the Appendix.

\subsection{Inferences under the common-equicorrelation model}

Under the common-equicorrelation model there is a common equicorrelation parameter $\rho$ and there are $k$ posssibly distinct variance parameters $\sigma_{i}^{2}$ among the independent studies. The posterior distribution $g$ of $\rho$ with respect to $\prod_{i}\left[\sigma_{i}^{-2} d \sigma_{i}^{2}\right] d \rho$ is given by

$$
g(\rho) \propto \frac{[1+(p-1) \rho]^{(p-1) n / 2}(1-\rho)^{n / 2}}{\prod_{i}\left\{[1+(p-1) \rho](p-1)\left(1-r_{i}\right)+(1-\rho)\left[1+(p-1) r_{i}\right]\right\}^{p n_{i} / 2}},
$$

where $\frac{-1}{p-1}<\rho<1, n=\sum_{i} n_{i}$ and $r_{i}$ is the sample equicorrelation coefficient

$$
r_{i}=\frac{2 \sum_{u<v}\left(S_{i}\right)_{u v}}{(p-1) \operatorname{tr} S_{i}}
$$

observed in the i-th study. The posterior moments of order $h=0,1, \ldots$ follow from numerically integrating $\rho^{h} g(\rho)$ in its (bounded) domain. The posterior mean and variance of $\sigma_{i}^{2}$ can be derived from the fact that $\sigma_{i}^{2}$ is the average of the $p$ eigenvalues $\lambda_{i 1}=\sigma_{i}^{2}[1+(p-1) \rho]$ and $\lambda_{i j}=\sigma_{i}^{2}(1-\rho), j=$ $2, \ldots, p$. The corresponding expressions for the posterior mean and variance of $\sigma_{i}^{2}$ are shown in the Appendix [(A.9),(A.10)]. 


\subsection{Inferences under the common-variance equicorrelation model.}

Under the common-variance equicorrelation model there is a common variance parameter $\sigma^{2}$ and there are posssibly $k$ distinct equicorrelation parameters $\rho_{i}$ among the collaborating studies. The joint posterior distribution $g$ of $\rho_{i}$ with respect to $\sigma^{-2} d \sigma^{2} \prod_{i} d \rho_{i}$ is given by

$$
g\left(\rho_{1}, \ldots, \rho_{k}\right) \propto \frac{\prod_{i}\left\{\left[1+(p-1) \rho_{i}\right]^{-n_{i} / 2}\left(1-\rho_{i}\right)^{-(p-1) n_{i} / 2}\right\}}{\left\{\sum_{i} \omega_{i}\left[(p-1) \frac{1-r_{i}}{1-\rho_{i}}+\frac{1+(p-1) r_{i}}{1+(p-1) \rho_{i}}\right]\right\}^{p n / 2}}
$$

where $\frac{-1}{p-1}<\rho_{i}<1, r_{i}$ is the sample equicorrelation coefficient observed in the i-th study, and $\omega_{i}=\operatorname{tr} A_{i} / \sum_{u} \operatorname{tr} A_{u}$. The posterior mixed-moments of order $h_{1}, \ldots, h_{k}$ follow from numerically evaluating the necessary versions of $\int \rho_{1}^{h_{1}} \ldots \rho_{k}^{h_{k}} g\left(\rho_{1}, \ldots, \rho_{k}\right) \prod_{i} d \rho_{i}$ in its (bounded) domain. Combined inferences for $\sigma^{2}$ based on the posterior distribution of $\lambda_{i 1}$ and $\lambda_{i 2}$ are discussed in the Appendix [(A.9), (A.10) and (A.12)].

\section{Analysis of the Keratometry Data}

In the present case there are $k=3$ experiments, each one consisting of observing $N_{i}=10$ curvature mappings $\mathbf{X}$ of the anterior reflective surface of a calibrated steel ball of different curvature. Repeated sample estimates of $\Sigma_{i}$ in our study, for several subregions of any given concentric field, have suggested an equicorrelation structure $\left(\sigma_{i}^{2}, \rho_{i}\right)$ with common equicorrelation $\rho=\rho_{i}$, and possibly different variances $\sigma_{i}^{2}$, corresponding to different refractive powers among steel balls (commonequicorrelation model). Consequently, the parameters of interest are $\left(\rho, \sigma_{1}, \sigma_{2}, \sigma_{3}\right)$, subject to $k=3$ and $N_{i}=10$. The data presented in Section 2 is based on observed curvatures at $p=4$ consecutive semimeridians $\{19,20,21,22\}$ at the aperture corresponding to field 18 . Note that the unrestricted number of covariance parameters within each experiment is $[p(p-1) / 2]+p=10$, compared with 2 , under the equicorrelation model.

Table 1 shows the component sample estimates $s_{i 1}, s_{i 2}, s_{i 3}, s_{i 4}$ of $\sigma_{i}$, the standard deviation of curvature measurements at semimeridians $\{19,20,21,22\}$, and sample estimates $r_{i}$ of the common equicorrelation $\rho$, for each calibrated steel balls $i=1,2,3$, based on samples of size $N_{i}=10$.

A standard numerical integration procedure showed that the the posterior estimate of $\rho$ based on $(4.1)$ is

$$
E\left(\rho \mid r_{1}, r_{2}, r_{3}\right)=0.939
$$

indicating a fairly large redundancy among the observed curvatures within the selected subset of 4 consecutive semimeridians. In particular, $E\left(\rho^{2} \mid r_{1}, r_{2}, r_{3}\right)=0.883$ and

$$
\operatorname{Sd}\left(\rho \mid r_{1}, r_{2}, r_{3}\right)=0.022
$$

is the posterior standard deviation of the combined estimate of $\rho$. Numerical evaluation of the posterior cumulative distribution of $\rho$ showed that $\rho$ falls in the interval $(0.88,0.97)$ with probability 0.96. Figure 1 illustrates the posterior distribution of $\rho$. Table 2 shows the posterior estimates of

$\sigma_{i}^{2}$ based on the posterior estimates of the eigenvalues of $\Sigma_{i}$. Expressions (A.9) and (A.10) were used to derive the results. The confidence limits for $\sigma_{i}^{2}$ shown in Table 2 are based on $1.5 \mathrm{Sd}$. 


\section{Summary and Discussion}

We have derived the combined posterior estimates of the equicorrelation parameter when sample covariance matrices $S_{1}, \ldots, S_{k}$ are available from several independent collaborating studies or experiments.

The combined posterior estimate of $\rho$ under the common-equicorrelation model depends on the available data only through the sample equicorrelations and their corresponding sample sizes. The posterior distribution of $\rho$ is given by (4.1). Standard numerical methods for integration in a given bounded interval can be used to determine the posterior moments of $\rho$. In applications with $p=2$ [e.g. vision data] the posterior distribution of $\rho$ becomes

$$
g(\rho) \propto \frac{\left(1-\rho^{2}\right)^{n / 2}}{\prod_{i}\left(1-\rho r_{i}\right)^{n_{i}}} .
$$

The joint posterior estimate of $\left(\rho_{1}, \ldots, \rho_{k}\right)$ under the equal- variance equicorrelation model depend of $r_{1}, \ldots, r_{k}$, their sample sizes and the weigths $\omega_{i}=\operatorname{tr} A_{i} / \sum_{u} \operatorname{tr} A_{u}$. When $p=2$ the joint posterior distribution (4.2) becomes

$$
g\left(\rho_{1}, \ldots, \rho_{k}\right) \propto \frac{\prod_{i}\left(1-\rho_{i}^{2}\right)^{-n_{i} / 2}}{\left\{\sum_{i} \omega_{i} \frac{1-\rho_{i} r_{i}}{1-\rho_{i}^{2}}\right\}^{n}} .
$$

Standard numerical methods for integration in a given bounded region can be used to determine the posterior mixed moments of $\rho_{1}, \ldots, \rho_{k}$.

The equal-variance equicorrelation model was also applied to the Keratometry data discussed earlier. Table 3 shows the posterior marginal estimates of $\rho_{i}$ and corresponding standard deviations and credibility limits. Figure 2 shows the cross-sections $g_{1}, g_{2}, g_{3}$ of the joint posterior distribution when all but one component are fixed and equal to the corresponding posterior marginal mean $\hat{\rho}_{i}$. For example, $g_{1}(\rho)=g\left(\rho, \widehat{\rho_{2}}, \widehat{\rho_{3}}\right)$

Combined maximum likelihood estimation of the equicorrelation structure was recently reported by the author (Viana 1994). The combined maximum likelihood estimate of $\rho$ under the common equicorrelation model $\left(\rho, \sigma_{1}, \ldots, \sigma_{k}\right)$ and the corresponding Bayesian estimates depend on the available data only through the sample intraclass correlations $r_{i} \quad i=1, \ldots, k$, and their relative sample sizes. The MLE of $\rho$ is the unique solution of a polynomial equation of degree $k$ in its domain. Standard numerical methods for determining the zeros of an equation in a given bounded interval can be used to determine the solution $\widehat{\rho}$. However, the estimated variance of $\widehat{\rho}$ is restricted to large-sample approximations. In contrast, the posterior moments of order $h=0,1, \ldots$ follow from numerically integrating $\rho^{h} g(\rho)$ in its (bounded) domain, as described earlier (4.1). Moreover, the exact posterior mean and variance of $\sigma_{i}^{2}$ can also be obtained directly from (A.9) and (A.10).

The joint MLE of $\left(\rho_{1}, \ldots, \rho_{k}\right)$ and corresponding Bayesian joint estimates under the equalvariance equicorrelation model $\left(\rho_{1}, \ldots, \rho_{k}, \sigma\right)$ depend on $r_{1}, \ldots, r_{k}$, their relative sample sizes and the relative weights $\omega_{i}=\operatorname{tr} A_{i} / \sum_{j=1}^{k} \operatorname{tr} A_{j}, i=1, \ldots, k$. The joint MLE is the numerical iterative solution of a system of polynomial equations and the corresponding variances are restricted to large-sample approximations, whereas, in contrast, the posterior mixed-moments 
of order $h_{1}, \ldots, h_{k}$ shown in (4.2) follow from numerically evaluating the necessary versions of $\int \rho_{1}^{h_{1}} \cdots \rho_{k}^{h_{k}} g\left(\rho_{1}, \ldots, \rho_{k}\right) \prod_{i} d \rho_{i}$ in its (bounded) domain. Consequently, when the sample sizes associated with the collaborating studies are small to moderate, the Bayesian method described above can be of particular advantage.

The estimates discussed above are based on the covariance estimates alone. However, when mean estimates are also considered, the additional assumption of symmetry $\mu_{i j}=m_{i}$ among the components of the underlying vector of means $\mu_{i}=\left(\mu_{i 1}, \ldots, \mu_{i p}\right)$ suggests the neccesary modifications to derive the combined estimates. Inferences about the two distinct eigenvalues remain essentially the same. The posterior distribution of $\lambda_{i 1}$ remains exactly the same, and the posterior distribution of $\lambda_{i 2}$ now has $v_{i 2}=(p-1) N_{i} / 2$ degrees of freedom and is based on $t_{i 2}+N_{i} \sum_{j}\left(\bar{x}_{i j}-\bar{x}_{i}\right)^{2} / 2$. The posterior distribution of $m_{i}$ can also be obtained and expressed in terms of a $t$ distribution. However, the posterior distribution of the equicorrelation coefficients $\rho_{i}$ cannot be shown in explicit form without the presence of the parameter $m_{i}$.

The posterior estimate of a common $\rho$ under the assumption of symmetric multivariate normal distributions is of particular interest to interpret familial data. Iterative maximum likelihood estimates have been considered by several authors including Donner and Koval (1980), Donner (1986), Donner and Bull (1983), Shoukri and Ward (1984), Srivastava (1984), Srivastava, Keen and Katapa (1988), Paul (1990), Paul and Barnwal (1990) and Gleser (1992). The posterior estimates of the equicorrelation coefficient derived in this paper can easily be aplicable to familial data as well. Of particular interest is the common- equicorrelation model based on $k$ independent sample covariance matrices of possibly different orders $p_{1}, \ldots, p_{k}$, to account for families with different number of members. In that case, the posterior distribution (4.1) of the common equicorrelation parameter becomes

$$
g(\rho) \propto \frac{(1-\rho)^{n / 2} \prod_{i}\left[1+\left(p_{i}-1\right) \rho\right]^{\left(p_{i}-1\right) n_{i} / 2}}{\prod_{i}\left\{\left[1+\left(p_{i}-1\right) \rho\right]\left(p_{i}-1\right)\left(1-r_{i}\right)+(1-\rho)\left[1+\left(p_{i}-1\right) r_{i}\right]\right\}^{p_{i} n_{i} / 2}},
$$

where $\min _{i}\left\{\frac{-1}{p_{i}-1}\right\}<\rho<1$. Although the present estimates are based on sample covariance data alone, the method applies to small samples and posterior credibility intervals can be obtained using standard methods of numerical intergration in bounded regions.

\section{A. Appendix}

Let $A_{i} \sim W_{p}\left(\Sigma_{i}, n_{i}\right), i=1, \ldots, k$ where $\Sigma_{i}$ has the equicorrelation form (3.1), with corresponding variance $\sigma_{i}^{2}$ and equicorrelation $\rho_{i}$. If $\Gamma$ is an orthogonal $p \times p$ matrix with first column constant and equal to $1 / \sqrt{p}$ [e.g. Press $(1972$, pages 13,14$)]$, then $A_{i}^{*}=\Gamma^{\prime} A_{i} \Gamma \sim W_{p}\left(D_{i}, n_{i}\right)$, where $D_{i}$ is a $p \times p$ diagonal matrix with diagonal entries

$$
\left(D_{i}\right)_{11}=\lambda_{i 1}=\sigma_{i}^{2}\left[1+(p-1) \rho_{i}\right], \quad\left(D_{i}\right)_{j j}=\lambda_{i 2}=\sigma_{i}^{2}\left(1-\rho_{i}\right), \quad j=2, \ldots, p .
$$

Consequently, the likelihood $\prod_{i} L\left(\sigma_{i}, \rho_{i}\right)$ can be expressed in terms of $\prod_{i=1}^{k} L\left(\lambda_{i 1}, \lambda_{i 2}\right)$, which factors orthogonally into $\prod_{i} P\left(q_{i 1} \mid \lambda_{i 1}\right) \prod_{j=2}^{p} P\left(q_{i j} \mid \lambda_{i 2}\right)$, where $P(\cdot \mid \cdot)$ indicates the appropriate density function corresponding to

$$
Q_{i 1}=\left(A_{i}^{*}\right)_{11}=\frac{\sum_{u, v}\left(A_{i}\right)_{u v}}{p} \sim W_{1}\left(\lambda_{i 1}, n_{i}\right), \quad Q_{i j}=\left(A_{i}^{*}\right)_{j j} \sim W_{1}\left(\lambda_{i 2}, n_{i}\right), \quad j=2, \ldots, p .
$$


Also note that $T_{i 1}=Q_{i 1} / 2$ can be expressed as

$$
T_{i 1}=\frac{N_{i}}{2}\left[1+(p-1) r_{i}\right] \operatorname{tr} S_{i} / p \sim W_{1}\left(\frac{\lambda_{i 1}}{2}, n_{i}\right),
$$

where $r_{i}$ is the conventional estimate of the equicorrelation coefficient $\rho_{i}$ (Olkin 1967, Olkin and Pratt 1958, Olkin and Siotani 1964),

$$
r_{i}=\frac{2 \sum_{u<v}\left(S_{i}\right)_{u v}}{(p-1) \operatorname{tr} S_{i}} .
$$

Also, $Q_{i 1^{\prime}}=\sum_{j=2}^{p} Q_{i j}=\operatorname{tr}\left(\Gamma^{\prime} A_{i} \Gamma\right)-\left(\Gamma^{\prime} A_{i} \Gamma\right)_{11}$, and since the matrix $\Gamma$ is orthogonal,

$$
T_{i 2}=\frac{1}{2}\left\{\operatorname{tr} A_{i}-\frac{1}{p} \sum_{u, v}\left(A_{i}\right)_{u v}\right\}=\frac{N_{i}}{2}(p-1)\left(1-r_{i}\right) \operatorname{tr} S_{i} / p \sim W_{1}\left(\frac{\lambda_{i 2}}{2},(p-1) n_{i}\right) .
$$

Inferences on $\lambda_{i 1}$. Let $v_{i 1}=n_{i} / 2$. From (A.2), the likelihood function based on $T_{i 1}$ can be expressed in terms of $\gamma_{1}\left(v_{i 1}, \lambda_{i 1}^{-1}\right)$, a gamma-1 distribution with parameters $v_{i 1}$ and $\lambda_{i 1}^{-1}$, so that the posterior distribution

$$
g\left(\lambda_{i 1} \mid t_{i 1}\right) \propto f_{\gamma 1}\left(t_{i 1} \mid v_{i 1}, \frac{1}{\lambda_{i 1}}\right) \frac{1}{\lambda_{i 1}}
$$

of $\lambda_{i 1}$ with respect to $\lambda_{i 1}^{-1}$ is an inverted-gamma-1 distribution $\gamma_{i n v, 1}(d, y)$ with parameters $v_{i 1}$ and $t_{i 1}$, [e.g. (Raiffa and Schlaifer 1968, pg. 227)]. In particular,

$$
E\left(\lambda_{i 1} \mid t_{i 1}\right)=\frac{t_{i 1}}{v_{i 1}-1}, \quad \operatorname{Var}\left(\lambda_{i 1} \mid t_{i 1}\right)=\frac{t_{i 1}^{2}}{\left(v_{i 1}-1\right)^{2}\left(v_{i 1}-2\right)} .
$$

Inferences on $\lambda_{i 2}$. Let $v_{i 2}=(p-1) n_{i} / 2$. The likelihood $L\left(\lambda_{i 2}\right)$ depends only on $Q_{i j} \sim$ $W_{1}\left(\lambda_{i 2}, n_{i}\right), \quad j=2, \ldots, p$, which, given $\lambda_{i 2}$, are independent. Similarly to $\lambda_{i 1}$, from (A.4) it follows that $T_{i 2} \mid \lambda_{i 2} \sim \gamma_{1}\left(v_{i 2}, \lambda_{i 2}^{-1}\right)$, and $\lambda_{i 2} \mid t_{i 2} \sim \gamma_{i n v, 1}\left(v_{i 2}, t_{i 2}\right)$, with respect to $p\left(\lambda_{i 2}\right) \propto \lambda_{i 2}^{-1}$, independent of the posterior distribution of $\lambda_{i 2}$, with respect to $\lambda_{i 1}^{-1} \lambda_{i 2}^{-1}$. In particular,

$$
E\left(\lambda_{i 2} \mid t_{i 2}\right)=\frac{t_{i 2}}{v_{i 2}-1}, \quad \operatorname{Var}\left(\lambda_{i 2} \mid t_{i 2}\right)=\frac{t_{i 2}^{2}}{\left(v_{i 2}-1\right)^{2}\left(v_{i 2}-2\right)} .
$$

Posterior distribution of $\rho_{i}$. Let $l_{i}=1-\rho_{i}, u_{i}=1+(p-1) \rho_{i}$. From (A.2) and (A.4) it follows that the posterior distribution of $\rho_{i}$ with respect to $\sigma_{i}^{-2}$ is

$$
g\left(\rho_{i} \mid t_{i 1}, t_{i 2}\right) \propto \int f_{\gamma_{1}}\left(t_{i 1} \mid v_{i 1}, \frac{1}{u_{i} \sigma_{i}^{2}}\right) f_{\gamma_{1}}\left(t_{i 2} \mid v_{i 2}, \frac{1}{l_{i} \sigma_{i}^{2}}\right) \frac{1}{\sigma_{i}^{2}} d \sigma_{i}^{2} \propto \frac{l_{i}^{v_{i 1}} u_{i}^{v_{i 2}}}{\left\{l_{i} t_{i 1}+u_{i} t_{i 2}\right\}^{v_{i 1}+v_{i 2}}} .
$$

Since $N_{i} t r S_{i} / 2=t_{i 1}+t_{i 2}$ can be factored out of $\left\{l_{i} t_{i 1}+u_{i} t_{i 2}\right\}$ and is determined by the data $\left\{t_{i 1}, t_{i 2}\right\}$ alone, the posterior distribution of $\rho_{i}$ can be expressed as

$$
g\left(\rho_{i} \mid t_{i 1}, t_{i 2}\right) \propto \frac{\left[1+(p-1) \rho_{i}\right]^{(p-1) n_{i} / 2}\left(1-\rho_{i}\right)^{n_{i} / 2}}{\left\{\left[1+(p-1) \rho_{i}\right](p-1)\left(1-r_{i}\right)+\left(1-\rho_{i}\right)\left[1+(p-1) r_{i}\right]\right\}^{p n_{i} / 2}} .
$$

Inferences on $\sigma_{i}^{2}$. To estimate $\sigma_{i}^{2}$ we use the fact that $p \sigma_{i}^{2}=\lambda_{i 1}+(p-1) \lambda_{i 2}$. Consequently, the posterior estimate of $\sigma_{i}^{2}$ with respect to $\left(\lambda_{i 1} \lambda_{i 2}\right)^{-1}$ is, from (A.6) and (A.7),

$$
E\left(\sigma_{i}^{2} \mid t_{i 1}, t_{i 2}\right)=\left[\frac{t_{i 1}}{v_{i 1}-1}+(p-1) \frac{t_{i 2}}{v_{i 2}-1}\right] / p
$$


Because $\lambda_{i 1}$ and $\lambda_{i 2}$ are independent, it follows that

$$
\operatorname{Var}\left(\sigma_{i}^{2} \mid t_{i 1}, t_{i 2}\right)=\left[\frac{t_{i 1}^{2}}{\left(v_{i 1}-1\right)^{2}\left(v_{i 1}-2\right)}+(p-1)^{2} \frac{t_{i 2}^{2}}{\left(v_{i 2}-1\right)^{2}\left(v_{i 2}-2\right)}\right] / p^{2} .
$$

Inferences under the common-equicorrelation model. Under the common-equicorrelation model there is a common value $l$ of $l_{i}=1-\rho_{i}$ and a common value $u$ of $u_{i}=1+(p-1) \rho_{i}$. Let $n=\sum n_{i}, v_{1}=\sum v_{i 1}=n / 2, v_{2}=\sum v_{i 2}=(p-1) n / 2, t_{1}=\left\{t_{11}, \ldots, t_{k 1}\right\}, t_{2}=\left\{t_{12}, \ldots, t_{k 2}\right\}$. The posterior distribution of the common equicorrelation $\rho$ given the joint data $t_{1}, t_{2}$ with respect to $\left[\prod \sigma_{i}^{2}\right]^{-1}$ is, from $(\mathrm{A} .8)$

$$
g\left(\rho \mid t_{1}, t_{2}\right) \propto \prod_{i} \int f_{\gamma_{1}}\left(t_{i 1} \mid v_{i 1}, \frac{1}{u \sigma_{i}^{2}}\right) f_{\gamma_{1}}\left(t_{i 2} \mid v_{i 2}, \frac{1}{l \sigma_{i}^{2}}\right) \frac{1}{\sigma_{i}^{2}} d \sigma_{i}^{2} \propto \frac{l^{v_{1}} u^{v_{2}}}{\prod_{i}\left\{l_{i} t_{i 1}+u_{i} t_{i 2}\right\}^{v_{i 1}+v_{i 2}}},
$$

which can be expressed as

$$
g\left(\rho \mid t_{1}, t_{2}\right) \propto \frac{[1+(p-1) \rho]^{(p-1) n / 2}(1-\rho)^{n / 2}}{\prod_{i}\left\{[1+(p-1) \rho](p-1)\left(1-r_{i}\right)+(1-\rho)\left[1+(p-1) r_{i}\right]\right\}^{p n_{i} / 2}} .
$$

Inferences for $\sigma_{i}^{2}$ based on the posterior distribution of $\lambda_{i 1}$ and $\lambda_{i 2}$ follow without change from (A.9) and (A.10).

Inferences under the common-variance equicorrelation model. Under the common-variance equicorrelation model there is a common value $\sigma$ of $\sigma_{i}^{2}$. The joint posterior distribution of $\rho_{1}, \ldots, \rho_{k}$ given $t_{1}, t_{2}$ with respect to $\sigma^{-2}$ follows from observing that (A.8) can be expressed as

$$
g\left(\rho_{i} \mid t_{i 1}, t_{i 2}\right) \propto \frac{l_{i}^{-v_{i 2}} u_{i}^{-v_{i 1}}}{\left\{\frac{t_{i 1}}{u_{i}}+\frac{t_{i 2}}{l_{i}}\right\}^{v_{i 1}+v_{i 2}}},
$$

which then generalizes to

$$
g\left(\rho_{1}, \ldots, \rho_{k} \mid t_{1}, t_{2}\right) \propto \int \prod_{i} f_{\gamma_{1}}\left(t_{i 1} \mid v_{i 1}, \frac{1}{u_{i} \sigma^{2}}\right) f_{\gamma_{1}}\left(t_{i 2} \mid v_{i 2}, \frac{1}{l_{i} \sigma^{2}}\right) \frac{1}{\sigma^{2}} d \sigma^{2} \propto \frac{\prod_{i} l_{i}^{v_{i 2}} u_{i}^{v_{i 1}}}{\sum_{i}\left\{\frac{t_{i 1}}{u_{i}}+\frac{t_{i 2}}{l_{i}}\right\}^{v_{1}+v_{2}}},
$$

and can be expressed as

$$
g\left(\rho_{1}, \ldots, \rho_{k} \mid t_{1}, t_{2}\right) \propto \frac{\prod_{i}\left\{\left[1+(p-1) \rho_{i}\right]^{-n_{i} / 2}\left(1-\rho_{i}\right)^{-(p-1) n_{i} / 2}\right\}}{\left\{\sum_{i} \frac{N_{i}}{2} \operatorname{tr} S_{i}\left[(p-1) \frac{1-r_{i}}{1-\rho_{i}}+\frac{1+(p-1) r_{i}}{1+(p-1) \rho_{i}}\right]\right\}^{p n / 2}},
$$

where $n=\sum_{i} n_{i}$. Note that in the above expression the coefficients $N_{i} \operatorname{tr} S_{i} / 2$ are determined by $t_{1}, t_{2}$ and can be replaced by the weights $\omega_{i}=\operatorname{tr} A_{i} / \sum_{u} \operatorname{tr} A_{u}$.

Combined inferences for $\sigma^{2}$ based on the posterior distribution of $\lambda_{i 1}$ and $\lambda_{i 2}$ follow from (A.9) and (A.10), observing that $\sigma^{2}=\sum_{i}\left[\lambda_{i 1}+(p-1) \lambda_{i 2}\right] / k p$ and that $\left\{\lambda_{i 1}, \lambda_{i 2}, i=1, \ldots, k\right\}$ are jointly independent, given $\left\{t_{1}, t_{2}\right\}$. Consequently, the mean combined estimate of $\sigma^{2}$ and its variance are

$$
E\left(\sigma^{2} \mid t_{1}, t_{2}\right)=\frac{1}{k} \sum_{i} E\left(\sigma_{i}^{2} \mid t_{i 1}, t_{i 2}\right), \quad \operatorname{Var}\left(\sigma^{2} \mid t_{1}, t_{2}\right)=\frac{1}{k^{2}} \sum_{i} \operatorname{Var}\left(\sigma_{i}^{2} \mid t_{i 1}, t_{i 2}\right) .
$$


Viana - August 31, 1994- Equicorrelation Coefficient

\section{References}

Donner, A. (1986), 'A review of inference procedures for the intraclass correlation coefficient in the one-way random effects model', International Statistical Review 54(1), 67-82.

Donner, A. and Bull, S. (1983), 'Inferences concerning a common intraclass correlation coefficient', Biometrics 39, 771-775.

Donner, A. and Koval, J. (1980), 'The estimation of intraclass correlation in the analysis of family data', Biometrics 36, 19-25.

Flury, B. (1988), Common Principal Components and Related Multivariate Models, Wiley, New York.

Gleser, L. J. (1992), 'A note on the analysis of familial data', Biometrika 79(2), 412-415.

Hage, S. E. (1976), 'The three-dimensional configuration of the cornea', Nouv. Rev. Optique pp. 205209.

Han, C. (1989), 'Combining tests for the correlation coefficient', The American Statistician 43(4), 211-215.

Hedges, L. and Olkin, I. (1985), Statistical Methods for Meta-Analysis, Academic Press, New York.

Klyce, S. and Wilson, S. (1989), 'Methods of analysis of corneal topography', Refractive and Corneal Surgery 5, 368-371.

Manly, B. and Rayner, J. (1987), 'The comparison of sample covariance matrices using likelihood ratio tests', Biometrika $\mathbf{7 4}(4)$, 841-847.

Olkin, I. (1967), Correlations revisted, in J. Stanley, ed., 'Improving Experiments: Design and Statistical Analysis', Seventh Annual Phi Delta Kappa Symposium on Educational Research, Rand and McNally, Chicago, pp. 102-128.

Olkin, I. and Pratt, J. (1958), 'Unbiased estimation of certain correlation coefficients', Annals of Mathematical Statistics 29, 201-211.

Olkin, I. and Siotani, M. (1964), Testing for the equality of correlation coefficients for various multivariate models, Technical report, Laboratory for Quantitative Research in Education, Stanford University.

Paul, S. R. (1990), 'Maximun likelihood estimation of intraclass correlation in the analysis of familial data: Estimating equation approach', Biometrika 77(3), 549-555.

Paul, S. R. and Barnwal, R. K. (1990), 'Maximun likelihood estimation and a c(alpha) test for a common intraclass correlation', The Statistician 39, 19-24.

Press, S. J. (1972), Applied Multivariate Analysis, Holt, Rinehart and Winston, New York.

Raiffa, H. and Schlaifer, R. (1968), Applied Statistical Decision Theory, M.I.T. Press, Cambridge. 
Rosner, B. (1982), 'Statistical methods in ophthalmology: An adjustment for the intraclass correlation between eyes', Biometrics 38, 105-114.

Rosner, B. (1984), 'Multivariate methods in ophthalmology with application to other paired-data situations', Biometrics 40, 1025-1035.

Shoukri, M. and Ward, R. (1984), 'On the estimation of the intraclass correlation', Communications in Statistics - Theory and Methods 13(10), 1239-1255.

Srivastava, M. (1984), 'Estimation of interclass correlations in familial data', Biometrika 71(1), 177185.

Srivastava, M., Keen, K. and Katapa, R. (1988), 'Estimation of interclass and intraclass correlations in multivariate familial data', Biometrics 44, 141-150.

Viana, M. A. G. (1980), 'Statistical methods for summarizing independent correlational results', Journal of Educational Statistics 5(1), 83-104.

Viana, M. A. G. (1994), 'Combined maximum likelihood estimates for the equicorrelation coefficient', Biometrics 50, ooo-ooo.

Viana, M. A. G., Olkin, I. and McMahon, T. (1993), 'Multivariate assessment of computer analyzed corneal topographers', J. Optical Society of America - A 10(8), 1826-1834.

Waring, G. (1989), 'Making sense of keratospeak ii: Proposed conventional terminology for corneal topography', Refractive and Corneal Surgery 5, 362-367. 
Figure 1: Posterior distribution of the equicorrelation coefficient under the common-equicorrelation model.

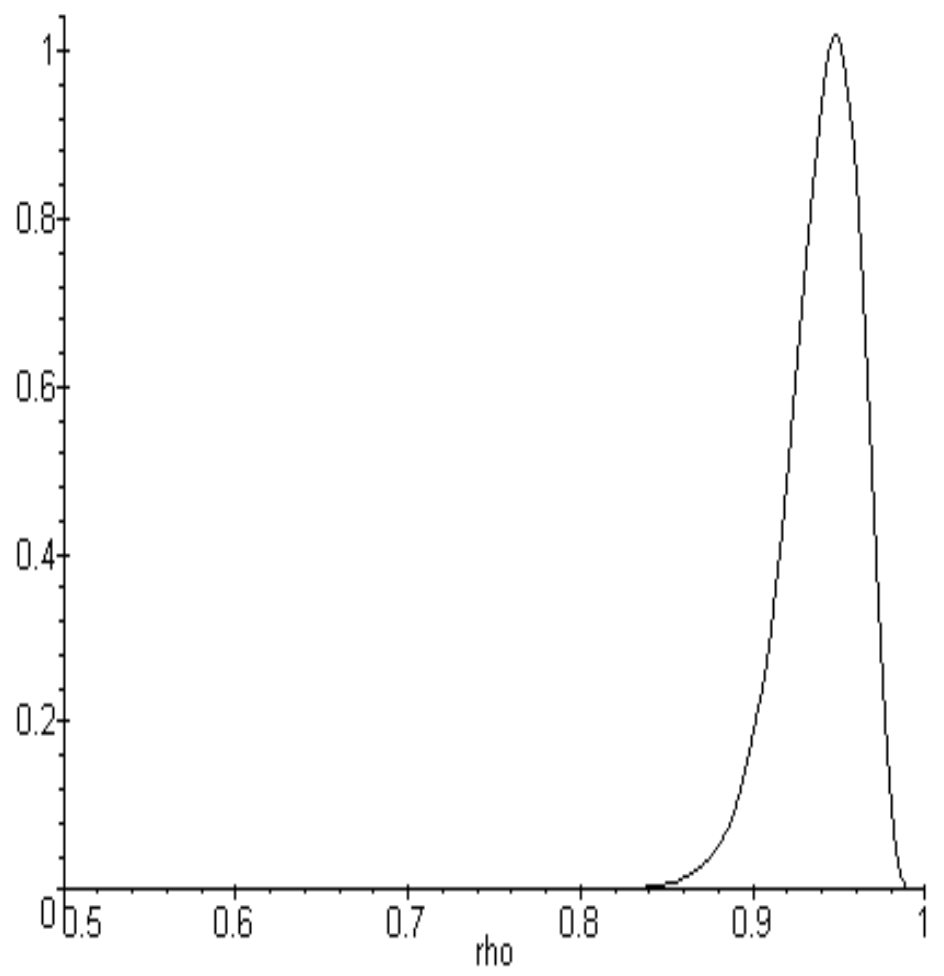


Figure 2: Cross-sections $g_{1}, g_{2}, g_{3}$ of the joint posterior distribution when all but one component are fixed and equal to the corresponding posterior marginal mean $\hat{\rho}_{i}$ of $\rho_{i}$.

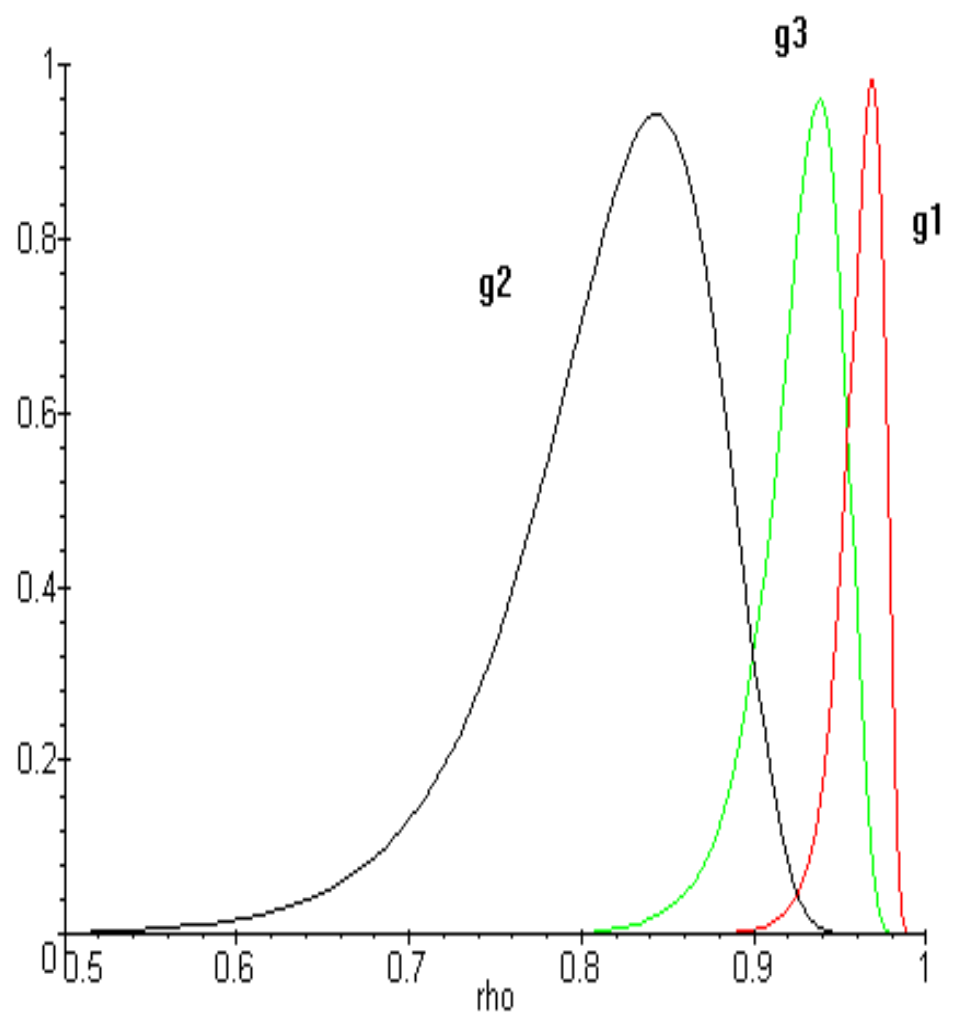


Table 1: Component sample estimates $s_{i 1}, s_{i 2}, s_{i 3}, s_{i 4}$ of $\sigma_{i}$, the standard deviation of curvature measurements, and sample estimate $r_{i}$ of the common equicorrelation $\rho$, for each calibrated steel ball $i=1,2,3$ at semimeridians $\{19,20,21,22\}$.

\begin{tabular}{rrrrrrrr}
\hline Ball [i] & Curvature & $N_{i}$ & $s_{i 1}$ & $s_{i 2}$ & $s_{i 3}$ & $s_{i 4}$ & $r_{i}$ \\
\hline 1 & 40.50 & 10 & .061 & .078 & .049 & .045 & .879 \\
2 & 42.50 & 10 & .098 & .138 & .089 & .086 & .814 \\
3 & 44.75 & 10 & .124 & .124 & .144 & .120 & .952 \\
\hline \hline
\end{tabular}

Table 2: Posterior estimates of $\sigma_{i}^{2}$ under the common-equicorrelation model, for each calibrated steel balls $i=1,2,3$ at semimeridians $\{19,20,21,22\}$.

\begin{tabular}{rrrrrrrrrrr}
\hline $\mathrm{i}$ & $t_{i 1}$ & $t_{i 2}$ & $E\left(\lambda_{i 1}\right.$ & $S d\left(\lambda_{i 1}\right)$ & $E\left(\lambda_{i 2}\right) 10^{4}$ & $S d\left(\lambda_{i 2}\right) 10^{4}$ & $E\left(\sigma_{i}^{2}\right)$ & $S d\left(\sigma_{i}^{2}\right)$ & $L_{i}$ & $U_{i}$ \\
\hline 1 & .0654 & .0065 & .0187 & .0118 & 5.2272 & 1.5414 & .0050 & .0029 & .0006 & .0095 \\
2 & .1893 & .0306 & .0540 & .0342 & 24.5520 & 7.2399 & .0153 & .0085 & .0025 & .0282 \\
3 & .3181 & .0118 & .0908 & .0574 & 9.5040 & 2.8025 & .0234 & .0143 & .0018 & .0449 \\
\hline \hline
\end{tabular}

Table 3: Posterior estimates of $\rho_{i}$ and corresponding credibility limits under the common-variance equicorrelation model, for each calibrated steel balls $i=1,2,3$ at semimeridians $\{19,20,21,22\}$.

\begin{tabular}{rrrrrrrr}
\hline Ball[i] & $r_{i}$ & $E\left(\rho_{i}\right)$ & $E\left(\rho_{i}^{2}\right)$ & $S d\left(\rho_{i}\right)$ & $L_{i}$ & $U_{i}$ & credibility \\
\hline 1 & .879 & .963 & .928 & .0113 & .93 & .98 & .92 \\
2 & .814 & .825 & .685 & .0661 & .66 & .91 & .92 \\
3 & .952 & .930 & .866 & .0331 & .89 & .96 & .81 \\
\hline \hline
\end{tabular}

